Neurobiology

\title{
Dopamine receptor disputes
}

\section{from J. M. Palacios}

'EstaBlisheD' ideas have a difficult time in neurobiology. Once again one of them, the question of the localization of dopamine receptors in the terminals of cortical neurones projecting to the striatum, is contested in a paper by J.M. Trugman et al. in this issue (Nature 323, 267; 1986). Brain receptors for the neurotransmitter dopamine, particularly those of the $\mathrm{D}_{2}$ type, mediate the action of many antipsychotic drugs and anti-parkinsonian agents. To know the exact cellular localization of dopamine receptors will help to elucidate the mechanisms whereby antipsychotic drugs exert their effects, and how these effects are channelled in the brain.

Recent progress in elucidating the site of dopamine receptors include their direct visualization in living human subjects using positron-emission tomography (Wagner, H.N. et al. Science 221, 1264; 1983). Although some brain areas, mainly basal ganglia nuclei such as the caudatus and putamen, are particularly enriched in $D_{2}$ receptors, we cannot yet determine in which cells, or where in the cells, they are located.

In the absence of probes to visualize directly dopamine receptors at the cellular level, biochemical assays of receptors, for example radioligand binding assays, are combined with the placement of neurotoxin or mechanical brain lesions (Schwarcz, R. et al. Nature 271, 766; 1978). Electrophysiologists also painstakingly try to determine the pre- and postsynaptic sites of action of dopamine. Their results are in general compatible with biochemical results (see, for example, Mercuri, N. et al. Brain Res. 358, 110; 1985). These kinds of experiments led to the generally accepted idea that dopamine $D_{2}$ receptors in the striatum are localized in: (1) the intrinsic neurones such as the interneurones that use acetylcholine as a messenger or the medium spiny neurones; (2) the axon terminals of a projection from the cortex that uses glutamate as transmitter (the cortico-striatal pathway); and (3) the terminals of the dopaminergic neurones themselves (presynaptic autoreceptors) whose cell bodies are located in the substantia nigra, a nucleus of the midbrain (see figure). This scheme is, however, based exclusively on circumstantial evidence and is subject to the many limitations of the techniques used.

This apparently well-established scheme is now contested by the paper of Trugman et al. in this issue. Using experimental procedures similar to those described above (a combination of specific lesions with binding assays) they conclude that most $\mathrm{D}_{2}$ receptors in the rat striatum are in the intrinsic striatal neurones and not equally divided between these cells and the terminals of the cortico-striatal pathway. The difference between these new results and previous ones arises from the use of quantitative receptor autoradiography, a technique (Young, W.S. \& Kuhar, M.J. Brain Res. 179, 255; 1979) allowing visualization of receptors at the level of resolution achievable with the

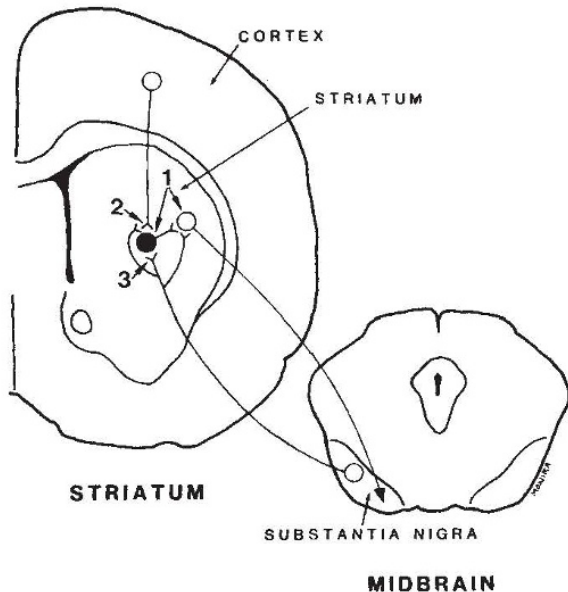

Proposed localizations of dopamine $\mathrm{D}_{2}$ receptors in the rat striatum. (1) Intrinsic neurones; (2) nerve terminals of the cortico-striatal pathway; and (3) nerve terminals of the nigrostriatal pathway. light microscope. Trugman et al. created lesions in the cortico-striatal pathway by suction of a small cortical area known to contain the cells of origin of the terminals in the striatum. These lesions produce degeneration in the striatum but do not affect the density of $D_{2}$ receptors. In contrast, when the toxin kainic acid is used to destroy the intrinsic neuronal cell bodies, but not afferent terminals, there is a large decrease of $\mathrm{D}_{2}$ receptor binding in the lesioned tissue.

How can the conflict with previous results be explained? Use of microscope methods allows the precise delimitation of the extent of the lesion and the calculation of the decrease of receptors in the area where neurones died and not in the unaffected remainder of the brain nucleus. This is difficult to control in biochemical and electrophysiological studies, and has a general bearing on the use of brain lesions in biochemical studies without appropriate histological control of the extent of the lesion.

Although provocative, the results in the paper of Trugman et al. do not solve the problem of the localization of dopamine receptors in the brain because of the lack of ultrastructural resolution. Only the development of probes such as antibodies directed against purified receptors or genetic probes will allow more precise identification of the cells that make dopamine receptors. Complete elucidation of the role of dopamine receptors in normal and psychotic brain function awaits further technical advances.

J. M. Palacios is in the Pharmaceutical Division at Sandoz Ltd, CH-4002 Basel, Switzerland.

\section{Parasitology}

\section{Improving prospects for a schistosomiasis vaccine}

\section{from S.R. Smithers}

AmID the expectations for the imminent development of a malaria vaccine, several laboratories have been working steadily towards a vaccine that will protect against another important human parasitic infection, schistosomiasis. Developments were reviewed at a recent meeting* and the consensus was that vaccination against schistosome infection in man is an attainable goal.

Man becomes infected through contact with fresh water containing infective cercariae shed from intermediate snail hosts. These penetrate exposed skin and rapidly transform to larval schistosomula that are physiologically adapted to live within the body. The schistosomula migrate

*Prospects for Immunological Intervention in Human Schisto somiasis Geneva, Switzerland 26-28 May 1986. through blood vessels to the lungs, then to the liver, where they mature into adult worms, and finally to the blood vessels of the intestine (Schistosoma mansoni) or bladder ( $S$. haematobium), where each male-female pair can produce several hundred eggs a day.

It is widely thought that the control of schistosomiasis by the drug praziquantel is so successful that no further measures are required. This view, however, has been sharply challenged. Reinfection can occur rapidly, particularly among children, who contribute most to parasite transmission. Treatment must therefore be repeated frequently, which increases costs and makes this form of control less attractive. Also, schistosomes resistant to an earlier drug, oxamniquine, have been recorded 